\title{
ERRATUM
}

\section{Volume 68, no. 5, 2002, Table of Contents (print version) and Author Index}

Table of Contents: Page numbers for the indicated articles should read as follows.

Metabolic Commensalism and Competition in a Two-Species Microbial Consortium

Double-Stranded RNA Mycovirus from Fusarium graminearum

Assessing the Role of Pseudomonas aeruginosa Surface-Active Gene Expression in Hexadecane Biodegradation in Sand

Effects of Amendment with Ferrihydrite and Gypsum on the Structure and Activity of Methanogenic Populations in Rice Field Soil

Quantitative Analysis of Cereulide, the Emetic Toxin of Bacillus cereus, Produced under Various Conditions

Alterations in Vibrio fischeri Motility Correlate with a Delay in Symbiosis Initiation and Are Associated with Additional Symbiotic Colonization Defects

Use of Recombinant Cellulose-Binding Domains of Trichoderma reesei Cellulase as a Selective

Immunocytochemical Marker for Cellulose in Protozoa

High-Density Microarray of Small-Subunit Ribosomal DNA Probes
Bjarke B. Christensen, Janus A. J.

Haagensen, Arne Heydorn, and

Søren Molin

Yeon-Mee Chu, Jae-Jin Jeon, SangJin Yea, Yong-Ho Kim, Sung-Hwan

Yun, Yin-Won Lee, and Kook-

Hyung Kim

P. A. Holden, M. G. LaMontagne,

A. K. Bruce, W. G. Miller, and S. E. Lindow

Tillmann Lueders and Michael W. Friedrich

Max M. Häggblom, Camelia

Apetroaie, Maria A. Andersson, an Mirja S. Salkinoja-Salonen

Deborah S. Millikan and Edward G. Ruby

Markus Linder, Jadwiga Winiecka-

Krusnell, and Ewert Linder

Kenneth H. Wilson, Wendy J.

Wilson, Jennifer L. Radosevich,

Todd Z. DeSantis, Vijay S.

Viswanathan, Thomas A.

Kuczmarski, and Gary L. Andersen
2503-2508

2495-2502

$2529-2534$

$2509-2518$

2484-2494

$2519-2528$

2535-2541

Author Index: Page numbers should read as follows. Christensen, Bjarke B., 2495; Haagensen, Janus A. J., 2495; Heydorn, Arne, 2495; Molin, Søren, 2495; Chu, Yeon-Mee, 2529; Jeon, Jae-Jin, 2529; Yea, Sang-Jin, 2529; Kim, Yong-Ho, 2529; Yun, Sung-Hwan, 2529; Lee, Yin-Won, 2529; Kim, Kook-Hyung, 2529; Holden, P. A., 2509; LaMontagne, M. G., 2509; Bruce, A. K., 2509; Miller, W. G., 2509; Lindow, S. E., 2509; Lueders, Tillmann, 2484; Friedrich, Michael W., 2484; Häggblom, Max M., 2479; Apetroaie, Camelia, 2479; Andersson, Maria A., 2479; Salkinoja-Salonen, Mirja S., 2479; Millikan, Deborah S., 2519; Ruby, Edward G., 2519; Linder, Markus, 2503; Winiecka-Krusnell, Jadwiga, 2503; Linder, Ewert, 2503; Wilson, Kenneth H., 2535; Wilson, Wendy J., 2535; Radosevich, Jennifer L., 2535; DeSantis, Todd Z., 2535; Viswanathan, Vijay S., 2535; Kuczmarski, Thomas A., 2535; Andersen, Gary L., 2535. 